\title{
Microendoscopic laminotomy versus conventional laminoplasty for cervical spondylotic myelopathy: 5-year follow-up study
}

\author{
Akihito Minamide, MD, PhD, ${ }^{1}$ Munehito Yoshida, MD, PhD, ${ }^{1}$ Andrew K. Simpson, MD, MHS, ${ }^{2}$ \\ Hiroshi Yamada, MD, PhD, ${ }^{1}$ Hiroshi Hashizume, MD, PhD, ${ }^{1}$ Yukihiro Nakagawa, MD, PhD, \\ Hiroshi Iwasaki, MD, PhD, ${ }^{1}$ Shunji Tsutsui, MD, PhD, ${ }^{1}$ Motohiro Okada, MD, PhD, ${ }^{1}$ \\ Masanari Takami, MD, PhD, ${ }^{1}$ and Shin-ichi Nakao, MD, PhD'
}

'Department of Orthopaedic Surgery, Wakayama Medical University, Wakayama, Japan; and ${ }^{2}$ Microendoscopic Spine Institute, Dallas, Texas

\begin{abstract}
OBJECTIVE The goal of this study was to characterize the long-term clinical and radiological results of articular segmental decompression surgery using endoscopy (cervical microendoscopic laminotomy [CMEL]) for cervical spondylotic myelopathy (CSM) and to compare outcomes to conventional expansive laminoplasty (ELAP).

METHODS Consecutive patients with CSM who required surgical treatment were enrolled. All enrolled patients $(n=$ 78) underwent CMEL or ELAP. All patients were followed postoperatively for more than 5 years. The preoperative and 5-year follow-up evaluations included neurological assessment (Japanese Orthopaedic Association [JOA] score), JOA recovery rates, axial neck pain (using a visual analog scale), the SF-36, and cervical sagittal alignment (C2-7 subaxial cervical angle).
\end{abstract}

RESULTS Sixty-one patients were included for analysis, 31 in the CMEL group and 30 in the ELAP group. The mean preoperative JOA score was 10.1 points in the CMEL group and 10.9 points in the ELAP group $(p>0.05)$. The JOA recovery rates were similar, $57.6 \%$ in the CMEL group and $55.4 \%$ in the ELAP group ( $p>0.05)$. The axial neck pain in the CMEL group was significantly lower than that in the ELAP group $(p<0.01)$. At the 5 -year follow-up, cervical alignment was more favorable in the CMEL group, with an average $2.6^{\circ}$ gain in lordosis (versus $1.2^{\circ}$ loss of lordosis in the ELAP group [ $<0.05]$ ) and lower incidence of postoperative kyphosis.

CONCLUSIONS CMEL is a novel, less invasive technique that allows for multilevel posterior cervical decompression for the treatment of CSM. This 5-year follow-up data demonstrates that after undergoing CMEL, patients have similar neurological outcomes to conventional laminoplasty, with significantly less postoperative axial pain and improved subaxial cervical lordosis when compared with their traditional ELAP counterparts.

https://thejns.org/doi/abs/10.3171/2017.2.SPINE16939

KEY WORDS cervical spine; cervical spondylotic myelopathy; clinical outcome; laminoplasty; endoscopic surgery; minimally invasive surgery; pincer mechanism

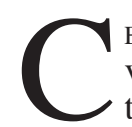
ERVICAL expansive laminoplasty (ELAP) for cervical myelopathy has been developed as a posterior decompression surgery that is reported to have favorable results. ${ }^{2,16,19}$ However, some problems have been reported after conventional ELAP due to damage to the cervical posterior soft tissues, including muscles and ligaments. Well-described potential complications of conventional laminoplasty include persistent axial pain, ${ }^{8,9,11}$ restriction of neck motion, and loss of lordotic curvature. Therefore, minimally invasive cervical decompressive techniques, which can allow for multilevel segmental decompression while preserving the paraspinal muscles and the posterior elements (such as the lamina, spinous processes, interspinous ligaments, and facet joints) have been developed. ${ }^{13,20,24}$ Minimally invasive laminotomy procedures using spinal endoscopy have become increasingly

ABBREVIATIONS CMEL = cervical microendoscopic laminotomy; CSM = cervical spondylotic myelopathy; ELAP = expansive laminoplasty; JOA = Japanese Orthopaedic Association; JOACMEQ = JOA Cervical Myelopathy Evaluation Questionnaire; OPLL = ossification of the posterior longitudinal ligament; VAS = visual analog scale. SUBMITTED August 6, 2016. ACCEPTED February 6, 2017.

INCLUDE WHEN CITING Published online July 14, 2017; DOI: 10.3171/2017.2.SPINE16939. 
popular for the treatment of a wide range of pathological conditions of the spine.,12,15,17,22 Endoscope-assisted posterior decompression surgery was derived from the microendoscopic discectomy technique developed by Foley and Smith. ${ }^{17}$ Over the last decade, microendoscopic techniques have been expanded to treat a multitude of conditions including lumbar spinal stenosis and, more recently, cervical conditions causing myelopathy and radiculopathy. Microendoscopic decompression techniques are advantageous because they involve smaller skin incisions, gentle and limited tissue dissection, and improved visualization compared with traditional tubular decompression methods, and the results obtained are equivalent to those with open techniques. One type of endoscope-assisted decompressive technique for cervical myelopathy is cervical microendoscopic laminotomy (CMEL). ${ }^{14,15}$ The compression of the cervical spinal cord in cervical spondylotic myelopathy (CSM) consists of a pincer mechanism due to a bulging disc and a hypertrophied ligamentum flavum. The concept of CMEL is to remove the dorsal compressive elements of the articular segment, including the ligamentum flavum and superior and inferior edge of the lamina, to provide safe relief of pressure off the involved spinal cord. CMEL preserves the interspinous, supraspinous, and paraspinal muscular insertions, which are removed during traditional ELAP techniques, and may provide some benefit with regard to maintaining lordosis and decreasing axial symptoms. Nonetheless, the long-term clinical benefits of CMEL in patients with CSM have not yet been elucidated. Therefore, the purpose of this study was to clarify the efficacy of posterior articular segment decompression by investigating the long-term clinical results of CMEL for patients with CSM, and to compare postoperative clinical and radiographic outcomes between conventional ELAP and CMEL techniques.

\section{Methods}

\section{Surgical Technique}

Following the induction of satisfactory general endotracheal anesthesia, the patient was secured in a Mayfield head holder, and was turned until prone. The patient's neck was fixed in a slightly flexed position to increase the interlaminar space, thus facilitating decompression. $\mathrm{C}$-arm fluoroscopy was used so that real-time lateral fluoroscopic images could be obtained. The operative surgeon generally stood on the side of the approach, with viewing monitors placed opposite him or her. Under lateral fluoroscopic guidance, the targeted level was marked on the side of the approach. A skin incision was made for the length of approximately $1.6 \mathrm{~cm}$, corresponding to the spinal level to be decompressed. The serial tubal dilators of the METRx endoscopic system (Medtronic Sofamor Danek), which was developed by Smith and Foley ${ }^{17}$ for lumbar disc herniation, were inserted through a small incision. After splitting into the paravertebral muscles, the tubular retractor lay on the lamina and facet joints, and was inclined in the direction of the intervertebral disc. Level and position of the tubular system were confirmed by lateral fluoroscopy. The inferior edge of the adjacent lamina and the medial edge of the facet complex were visualized.
The technique consisted of bilateral decompression using a unilateral approach (Fig. 1A). Endoscope-assisted laminotomy was performed up to the cephalad insertion of the attachment of the ligamentum flavum using a long, curved, high-speed drill with endoscopic bit (e.g., Midas Rex; Medtronic). The superior attachment of the ligamentum flavum was exposed, and the procedure was then continued to the superior portion of the inferior lamina. The inferior attachment of the ligamentum flavum was then similarly exposed. After the base of the spinous process was drilled, the laminotomy on the contralateral side was performed by drilling and tunneling the lamina from the spinal canal side. It is important to perform the contralateral decompression without removing the ligamentum flavum, to protect the spinal cord. When the floated ligamentum flavum (Fig. 1B) was completely removed, dural pulsation was observed (Fig. 1C). When endoscopic laminotomy was needed for the adjacent level, the tubular retractor was inclined either cranially or caudally and the decompression was then similarly performed (Fig. 1D).

\section{Patient Population}

This study was approved by the IRB of Wakayama Medical University. Between January 2005 and December 2010, consecutive patients were enrolled in this study if they had symptoms of cervical myelopathy and evidence of associated spinal stenosis due to cervical spondylosis. Informed consent was obtained for inclusion in the study. Inclusion criteria were as follows: 1) neurological signs consistent with cervical myelopathy, such as upper- or lower-extremity clumsiness, numbness of the upper and/or lower extremities, and gait disturbance/imbalance; and 2) spinal cord compression demonstrated by MRI, myelography, and postmyelography CT. The criteria for exclusion were trauma, tumor, severe ossification of the posterior longitudinal ligament (OPLL), rheumatoid arthritis, infection, destructive spondyloarthropathies, and other combined spinal lesions. The remaining patients were included in this cohort study. Subsequently, all enrolled patients underwent CMEL ${ }^{15}$ or conventional ELAP (French-door ${ }^{24}$ or open-door type ${ }^{7}$ ) at the authors' institution. Surgeries were performed by 4 spine surgeons. The 2 spine surgeons who were authorized by the Japanese Orthopaedic Association (JOA) as endoscopic spine surgeons performed the CMEL surgery, whereas other surgeons performed the ELAP surgery. Patients were divided into 2 surgical groups, consisting of the CMEL or ELAP group, according to the turn that an operation was required (in the outpatient clinic). Postoperatively, the use of a neck brace was left to the patients' discretion.

\section{Clinical Outcomes}

The patients' preoperative and 5-year follow-up functional outcomes were evaluated using the JOA scoring system for cervical myelopathy (JOA score, full score = 17 points), ${ }^{23}$ Cervical Myelopathy Evaluation Questionnaire (JOACMEQ), ${ }^{4}$ SF-36 $6^{3}$ survey, and visual analog scale (VAS) for the assessment of axial neck pain. Lateral radiographs were taken in the neutral position preoperatively and at 5 years postoperatively. The lordotic angle 


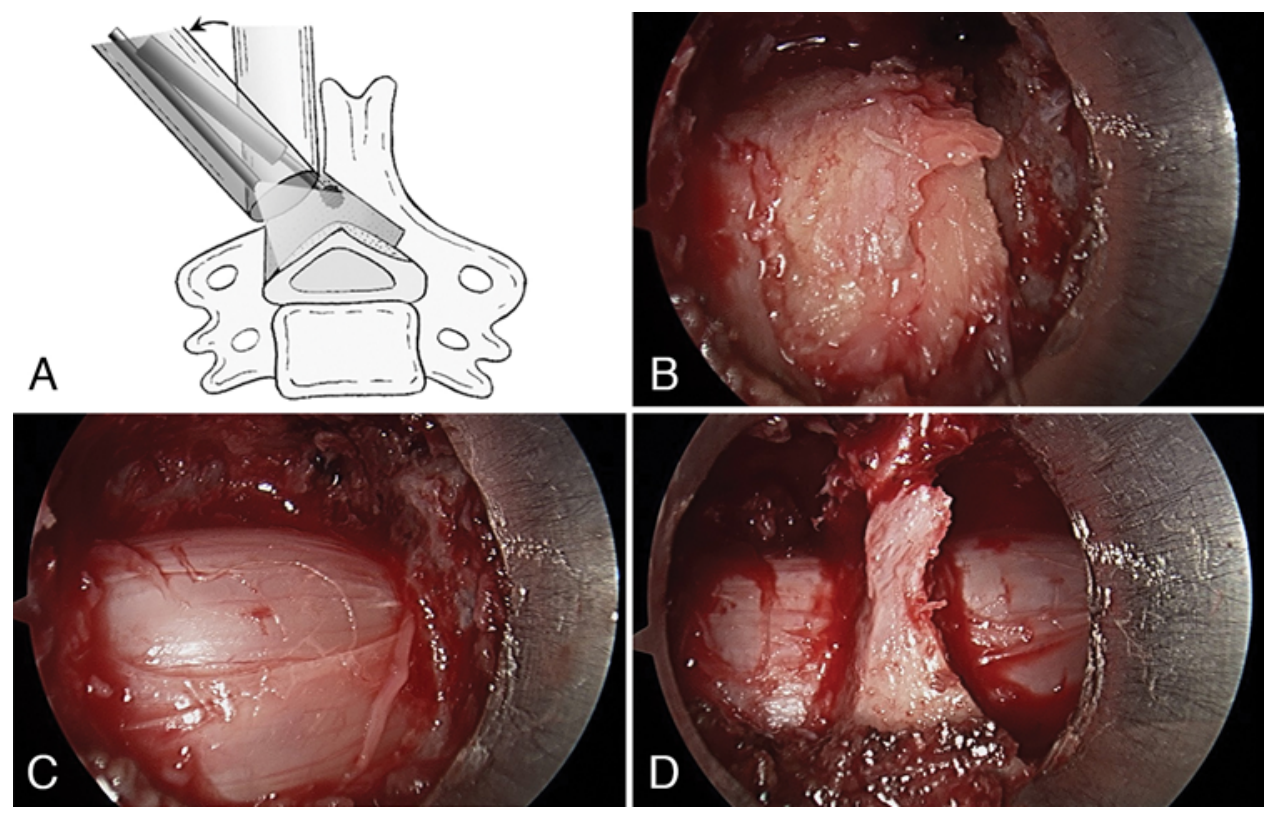

FIG. 1. CMEL is performed for CSM. A: The decompression surgery is performed using a high-speed air drill. The hemilaminectomy is performed on the approaching side, and then the laminotomy on the contralateral side is completed. The concept of CMEL is to remove the elements of the articular segment, such as the ligamentum flavum and superior or inferior edge of the lamina. Copyright Akihito Minamide. Published with permission. B: When the spinal cord is completely decompressed in the element of the articular segment, the floated ligamentum flavum is observed. C: By removing the ligamentum flavum, the dural pulsation is observed. D: For multiple levels, the tubular retractor is inclined cranially or caudally, and the above procedure is repeated. Figure is available in color online only.

was calculated by measuring between the C-2 inferior endplate and C-7 superior endplates at the neutral position using Cobb's method. The change in alignment was determined by the difference between subaxial alignment at the preoperative and 5-year postoperative radiographs. The results obtained were filed in the patients' charts and were not the basis for any decision making. Data collection was performed by independent reviewers (H.H. and S.N.). Clinical outcomes were based on the recovery rates calculated from the JOA score. The recovery rate was calculated as follows: recovery rate $=100 \times$ (postoperative JOA score - preoperative JOA score)/(17 - preoperative JOA score). The data regarding intraoperative, perioperative, and postoperative complications were retrieved from independent review of the medical charts.

\section{Statistical Analysis}

All parameters were analyzed statistically. The Student t-test was used to compare preoperative and postoperative JOA scores, recovery rates, SF-36 scores, and VAS scores between the CMEL and ELAP groups. The subaxial cervical lordosis (C2-7 Cobb angle) on lateral radiography was also compared using the Student t-test. All statistical analyses were performed using JMP (version 11, SAS Inc.), with the level of significance set at $\mathrm{p}<0.05$.

\section{Results}

Seventy-eight patients were included in the study, including 50 men and 28 women, with a mean age of 65.5 years (range $46-85$ years). Nine patients were lost to follow-up; thus, the follow-up rate was $88.5 \%$ (69 of 78 pa- tients). An additional 8 patients were excluded because of death, dementia, or concurrent symptomatic lumbar spinal stenosis. Thus, 61 patients completed the 5-year follow-up and were included for data analysis; there were 31 patients in the CMEL group and 30 in the ELAP group (Table 1).

There were no significant differences in sex, age, preoperative JOA scores, and recovery rate of JOA score between the CMEL and ELAP groups $(p>0.05$; Tables 1 and 2). In addition, there were significant improvements between preoperative and final JOA scores in both groups $(\mathrm{p}<0.05)$.

TABLE 1. Characteristics of patients who completed the 5-year follow-up, according to operative group

\begin{tabular}{lccc}
\hline \multicolumn{1}{c}{ Variable } & CMEL* $^{*}$ & ELAP* & $\begin{array}{c}p \\
\text { Value† }\end{array}$ \\
\hline No. of patients & 31 & 30 & \\
$\quad$ Male/female ratio & $19: 12$ & $19: 11$ & 0.95 \\
$\quad$ Age (yrs) & $59.0 \pm 14.4$ & $62.5 \pm 13.8$ & 0.34 \\
Preop JOA score & $10.1 \pm 2.6$ & $10.9 \pm 2.5$ & 0.24 \\
Preop VAS score§ & $51.5 \pm 30.0$ & $56.0 \pm 30.7$ & 0.44 \\
No. of surgically affected levels & $2.0 \pm 0.8$ & $3.9 \pm 0.3$ & $<0.0001$ \\
Op time (mins) & $118 \pm 35$ & $172 \pm 38$ & $<0.0001$ \\
Blood loss (ml) & $32.8 \pm 36.1$ & $232.6 \pm 229.6$ & $<0.0001$ \\
1-day postop creatine kinase & $94.9 \pm 35.0$ & $403.3 \pm 213.3$ & $<0.0001$ \\
$\quad$ & & & \\
\hline (IU/L) & & & \\
\hline
\end{tabular}

\footnotetext{
* Data given as the mean \pm SD unless otherwise indicated.

+ Values $<0.05$ are statistically significant.

$\ddagger$ JOA scoring system; full score $=17$ points.

$\S$ VAS score for axial symptoms; full scale $=100 \mathrm{~mm}$.
} 
TABLE 2. Clinical outcomes by procedure at 5-year follow-up

\begin{tabular}{lccc}
\hline \multicolumn{1}{c}{ Scale } & CMEL $^{*}$ & ELAP $^{*}$ & $p$ Value \\
\hline JOA & $14.0 \pm 2.0$ & $13.9 \pm 2.4$ & 0.75 \\
JOA recovery rate & $57.6 \pm 17.6$ & $55.4 \pm 25.1$ & 0.69 \\
VAS & $19.5 \pm 20.1$ & $43.6 \pm 29.2$ & $<0.01$ \\
\hline
\end{tabular}

* Data given as the mean \pm SD.

Regarding perioperative complications, 2 patients in the CMEL group and 1 patient in the ELAP group experienced C-5 nerve root palsy postoperatively. The 2 patients in the CMEL group had the palsy on the approach side. All patients improved as a result of conservative treatment with a Philadelphia collar. The developmental nerve root palsy appeared several days following surgery. The tethering effect on the nerve root induced by excessive posterior shift of the spinal cord after decompression was the additional hypothesized cause. The VAS score for axial neck symptoms at the final follow-up evaluation was significantly lower in the CMEL group than the ELAP group. Regarding the JOACMEQ and SF-36 scores, there were no significant differences in any subscale at the 5-year evaluation between groups ( $\mathrm{p}>0.05$; Figs. $2-4$ ).

In the CMEL group, the mean lordotic angle was $12.7^{\circ}$ preoperatively and $14.9^{\circ}$ at the 5-year follow-up; in the ELAP group, the mean lordotic angle was $10.0^{\circ}$ preoperatively and $7.9^{\circ}$ at the 5-year follow-up (Table 3). The difference between lordotic angles preoperatively and at 5 years was $2.6^{\circ}$ in the CMEL group, indicating a gain in cervical lordosis, and $-1.2^{\circ}$ in the ELAP group, indicating a loss of cervical lordosis. There were significant betweengroups differences with respect to the mean lordotic angle at the 5-year follow-up and the difference between preoperative and 5-year follow-up values $(\mathrm{p}<0.05)$. With regard to changes in the alignment of the lateral neutral position, 2 patients in the CMEL group and 4 patients in the ELAP group presented with preoperative lordosis that changed to kyphosis at the 5-year follow-up. Seven patients (22.6\%)

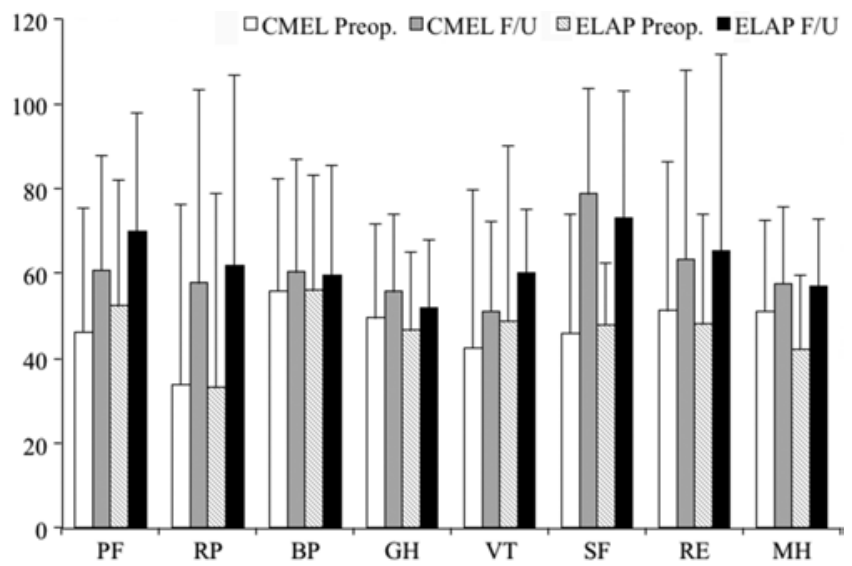

FIG. 2. Evaluation of SF-36 scores at the 5 -year follow-up (F/U). All scales in the CMEL group were equal to those of the ELAP group. BP = bodily pain; $\mathrm{GH}=$ general health perceptions; $\mathrm{MH}=$ mental health; $\mathrm{PF}$ = physical functioning; $\mathrm{RE}=$ role emotional; $\mathrm{RP}=$ role physical; $\mathrm{SF}=$ social functioning; $V T$ = vitality.

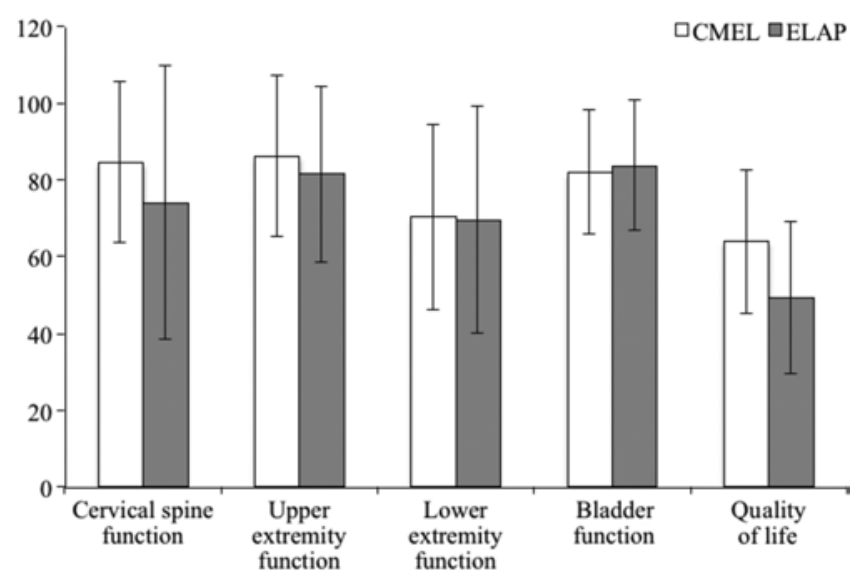

FIG. 3. Comparison of scores on the JOACMEQ between groups. There were no significant differences in any subscale of the JOACMEQ between groups.

in the CMEL group and 2 patients $(6.7 \%)$ in the ELAP group exhibited an increase in lordotic angle greater than $10^{\circ}$. This result suggests that CMEL maintained lordosis better than ELAP.

\section{Discussion}

Conventional ELAP techniques provide surgeons with a powerful tool for decompression of multilevel cervical stenosis, while maintaining intersegmental motion and avoiding the significant loss of clinical motion that results from multilevel fusions. However, the complications of these traditional laminoplasty techniques, including persistent axial symptoms and neck pain, as well as loss of subaxial cervical lordosis and resultant sagittal imbalance, are well described. There are a multitude of reports demonstrating persistent axial symptoms following ELAP, $, 8,9,11$ and the frequency of clinically significant postoperative neck pain is nearly 3 times that of anterior cervical fusion..$^{9}$ A number of surgical technique modifications, as well as programs for early neck mobilization, have been developed for conventional cervical ELAP to prevent these types of morbidities, but the issues of postoperative axial symptoms and loss of lordosis have remained. ${ }^{6,10,16,}$ ${ }^{18,21}$ Without question, intraoperative damage to cervical posterior soft tissues, including muscles and ligaments, are at least somewhat responsible for many of these problems.

We have been using CMEL as a less invasive surgical strategy for posterior cervical decompression in cervical myelopathy. ${ }^{15}$ The principal benefit of the endoscopeassisted procedure is that it minimizes disruption of the posterior structures (Fig. 5), while still allowing for thorough segmental decompression. Prior to this study, the longer-term clinical outcomes with regard to neurological recovery, axial symptoms, and radiographic parameters for CMEL had not yet been elucidated. The purpose of this study was to characterize these outcomes for microendoscopic laminotomy, and to compare them to those for conventional ELAP.

The primary goal in treating multilevel CSM is to arrest neurological deterioration and, in many cases, to improve a patient's neurological status. Therefore, as we develop less 

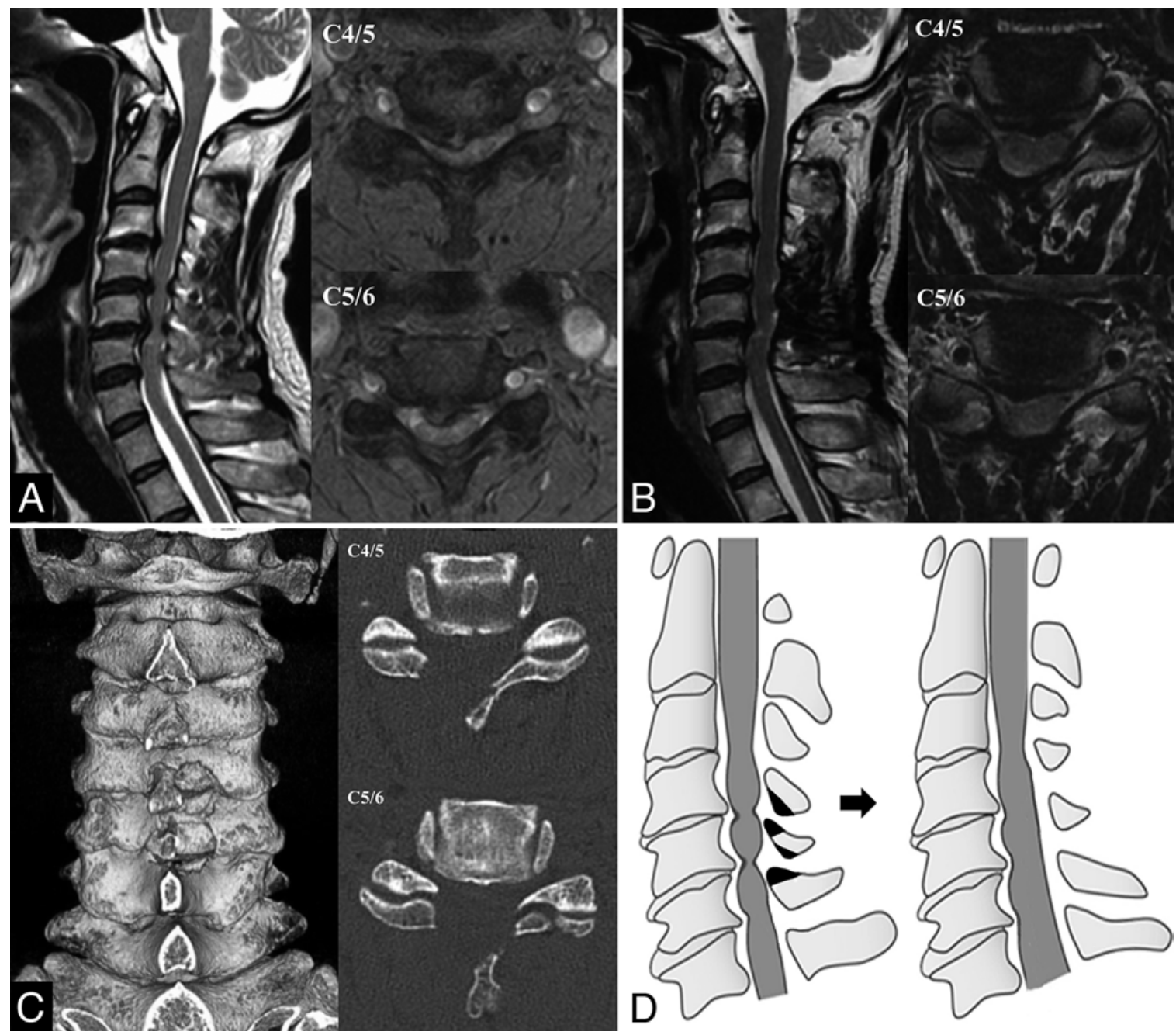

FIG. 4. Illustrative case. A 53-year-old woman with CSM presented with clumsiness, numbness in the bilateral hands, and spastic gait. A: MRI showing spinal cord compression with spondylosis at the C4-5 and C5-6 levels, with particularly severe spinal cord compression due to intervertebral disc herniation at $\mathrm{C} 4-5$. The patient underwent CMEL from the $\mathrm{C}-4$ to $\mathrm{C}-6$ levels. The JOA score improved from 13 points preoperatively to 16 points 5 years postoperatively. B and C: MRI (B) and CT (C) scans obtained 1 year postoperatively showing the success of spinal cord decompression. Axial images also show the successfully decompressed spinal cord at each level. In the unilateral approach, the laminotomies are performed at each level. D: The CMEL is performed to remove the elements of the articular segment, such as the ligamentum flavum and superior or inferior edge of the lamina. Panel D: Copyright Akihito Minamide. Published with permission.

invasive strategies for the treatment of spine conditions, it is paramount that we not compromise our ability to safely address patients' neurological and functional measures. In our study, patients demonstrated similar preoperative neurological status, as measured by JOA score, and both groups undergoing conventional laminoplasty and CMEL had similar neurological recovery rates; thus, both ELAP and CMEL similarly achieved the primary goal of neurological recovery in patients with CSM.

Conventional laminoplasty techniques have been well studied, and the most commonly cited complications involve postoperative axial neck pain and loss of lordosis. ${ }^{6,10}$, ${ }^{18,21}$ These parameters were specifically addressed in this study, and compared between ELAP and CMEL. The VAS score for axial neck symptoms at the final follow-up was significantly lower in the CMEL group than the ELAP group (19.5 vs 43.6, $\mathrm{p}<0.01$; Table 2). This is a rather dramatic difference in axial symptoms when comparing endoscopic techniques to more traditional laminoplasty. With regard to radiographic parameters, CMEL also dem-

TABLE 3. Subaxial cervical lordosis measured between the $\mathbf{C}-2$ and $\mathrm{C}-7$ angle at the neutral position using Cobb's method on lateral radiographs

\begin{tabular}{lcrl}
\hline \multicolumn{1}{c}{ Variable } & CMEL & \multicolumn{1}{c}{ ELAP } & p Value \\
\hline Mean preop angle $\pm \mathrm{SD}\left({ }^{\circ}\right)$ & $12.7 \pm 15.1$ & $10.0 \pm 8.3$ & 0.45 \\
Mean 5-yr follow-up angle $\pm \mathrm{SD}\left({ }^{\circ}\right)$ & $14.9 \pm 15.8$ & $7.9 \pm 9.5$ & 0.042 \\
Difference btwn preop \& 5 $\mathrm{yrs}^{*}$ & $2.6 \pm 7.7$ & $-1.2 \pm 5.0$ & 0.031 \\
\hline
\end{tabular}

* Difference between subaxial cervical lordosis on preoperative and 5 -year postoperative radiographs. 


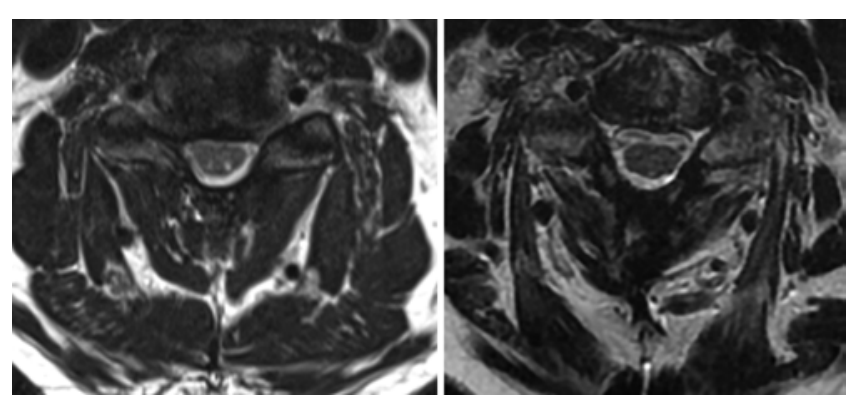

FIG. 5. Influence of surgical techniques on soft tissues. The influence of endoscopic surgery (left) on the soft tissues differs substantially from that of conventional laminoplasty (right). Axial MRI shows no changes of fatty degeneration in the paravertebral muscles after CMEL.

onstrated favorable results compared with ELAP, with 5-year postoperative radiographs demonstrating an average $2.6^{\circ}$ gain in subaxial cervical lordosis after CMEL, versus a $1.2^{\circ}$ loss of lordosis after ELAP $(p=0.03)$. There were twice as many patients in the ELAP group who developed kyphosis postoperatively. The preservation of these dorsal structures, including the paraspinal musculature and ligamentum flavum insertions, ought to limit functional loss of the posterior tension band and associated kyphosis, as well as limit axial symptoms from tissue damage. There is literature on both the cervical and lumbar spine to demonstrate improvement in lordosis after decompression surgery alone, which is theorized to be secondary to postural changes. Specifically, patients with stenosis often open the dimensions of the spinal canal by flexion, which decreases the posterior pincer mechanism and contributes to a more flexed position on preoperative neutral radiographic evaluation. Hence, they may stand in more postural extension once these compressive structures are removed. Given the ever-increasing amount of evidence regarding the importance of cervical sagittal alignment and its effect on both functional and neurological outcomes, the improved ability of CMEL to maintain lordosis may prove even more important as we follow these patients postoperatively for longer periods.

This study has several important limitations. First, all procedures were performed by a limited number of surgeons at a high-volume center for the treatment of CSM. We have tremendous experience with microendoscopic techniques, and while this does provide some standardization of technique, they may not be generalizable to a larger population of surgeons. Because of the skill of the operator of endoscopic spinal surgery, the treatment modality for some patients may be determined by the surgeon's individual decision making, so inherent differences between groups cannot be excluded. However, they were a relatively matched cohort as preoperative age, sex, sagittal alignment, and JOA scores were similar between the 2 groups. In the future it will be necessary to determine whether the CMEL surgery is indicated for all conditions of CSM pathology, including spinal instability. Additionally, in future endeavors it would be interesting to investigate how CMEL would compare with ventral procedures for CSM, although we believed that direct comparison with a similar dorsal intervention such as laminoplasty was the most appropriate initial evaluation.
CMEL is a novel, less invasive technique that allows for multilevel posterior cervical decompression for the treatment of CSM. Our study demonstrates that neurological outcomes are similar to conventional laminoplasty techniques. Moreover, 5-year follow-up data demonstrate that after CMEL, patients have far less postoperative axial pain and improved subaxial cervical lordosis when compared with their traditional laminoplasty counterparts. CMEL is fundamentally different from conventional laminoplasty, as the former decompresses the pincer mechanism at the level of the disc space, while the latter actually opens the bony aspect of the vertebral canal in addition to decompressing the disc space. Thus, CMEL has a more limited role and cannot be used in patients with significant congenital stenosis or retrovertebral ventral compression, such as OPLL, where compression exists at more than just the level of the disc space. Hatta et al..$^{5}$ reported that the outcome of posterior decompression surgery for multisegmental CSM was not correlated with the magnitude of postoperative posterior shift of the spinal cord. Posterior decompression of the articular segment may be enough in patients with spondylotic-type stenosis. In this study, the number of surgical disc levels was significantly different between the 2 groups, although there was no difference in the surgical selection criteria for the patients with CSM. Based on the general concept of a posterior shift of the spinal cord with ELAP, this procedure was performed at C3-5, C3-6, or C3-7, even in patients with CSM in whom the main lesion responsible for the condition was located at 1 or 2 disc levels. For example, a patient with CSM and 1 lesion at the C4-5 level underwent ELAP surgery from C-3 to C-5, whereas a patient with CSM and 2 lesions at the C4-5 and C5-6 levels underwent ELAP surgery from C-3 to C-6. With CMEL, however, surgery was performed only at the affected levels. Due to this inherent difference between these treatment modalities, the average number of levels included in ELAP patients was more than that included in CMEL patients. Thus, one could argue that some of the relative improvement in outcome measures was a result of the ability of CMEL to address solely the affected levels.

\section{Conclusions}

This work demonstrates that, in the appropriately selected patient with CSM secondary to typical stenosis patterns at the level of the disc space and anatomical parameters favorable for dorsal decompression techniques, CMEL represents an effective technique with long-term neurological outcomes that are similar to conventional laminoplasty methods, with less tissue disruption, which translates into significantly less postoperative neck pain and improved postoperative sagittal alignment.

\section{References}

1. Adamson TE: Microendoscopic posterior cervical laminoforaminotomy for unilateral radiculopathy: results of a new technique in 100 cases. J Neurosurg 95 (1 Suppl):51-57, 2001

2. Chiba K, Ogawa Y, Ishii K, Takaishi H, Nakamura M, Maruiwa $\mathrm{H}$, et al: Long-term results of expansive open-door laminoplasty for cervical myelopathy-average 14-year follow-up study. Spine (Phila Pa 1976) 31:2998-3005, 2006 
3. Fukuhara S, Bito S, Green J, Hsiao A, Kurokawa K: Translation, adaptation, and validation of the SF-36 Health Survey for use in Japan. J Clin Epidemiol 51:1037-1044, 1998

4. Fukui M, Chiba K, Kawakami M, Kikuchi S, Konno S, Miyamoto M, et al: Japanese Orthopaedic Association Cervical Myelopathy Evaluation Questionnaire (JOACMEQ): part 4. Establishment of equations for severity scores. Subcommittee on low back pain and cervical myelopathy, evaluation of the clinical outcome committee of the Japanese Orthopaedic Association. J Orthop Sci 13:25-31, 2008

5. Hatta Y, Shiraishi T, Hase H, Yato Y, Ueda S, Mikami Y, et al: Is posterior spinal cord shifting by extensive posterior decompression clinically significant for multisegmental cervical spondylotic myelopathy? Spine (Phila Pa 1976) 30:2414-2419, 2005

6. Heller JG, Edwards CC II, Murakami H, Rodts GE: Laminoplasty versus laminectomy and fusion for multilevel cervical myelopathy: an independent matched cohort analysis. Spine (Phila Pa 1976) 26:1330-1336, 2001

7. Hirabayashi K, Watanabe K, Wakano K, Suzuki N, Satomi K, Ishii Y: Expansive open-door laminoplasty for cervical spinal stenotic myelopathy. Spine (Phila Pa 1976) 8:693699, 1983

8. Hosono N, Sakaura H, Mukai Y, Fujii R, Yoshikawa H: C3-6 laminoplasty takes over C3-7 laminoplasty with significantly lower incidence of axial neck pain. Eur Spine J 15:13751379,2006

9. Hosono N, Yonenobu K, Ono K: Neck and shoulder pain after laminoplasty. A noticeable complication. Spine (Phila Pa 1976) 21:1969-1973, 1996

10. Iizuka H, Nakajima T, Iizuka Y, Sorimachi Y, Ara T, Nishinome M, et al: Cervical malalignment after laminoplasty: relationship to deep extensor musculature of the cervical spine and neurological outcome. J Neurosurg Spine 7:610-614, 2007

11. Kawaguchi Y, Matsui H, Ishihara H, Gejo R, Yoshino O: Axial symptoms after en bloc cervical laminoplasty. J Spinal Disord 12:392-395, 1999

12. Khoo LT, Fessler RG: Microendoscopic decompressive laminotomy for the treatment of lumbar stenosis. Neurosurgery 51 (5 Suppl):S146-S154, 2002

13. Liu J, Ebraheim NA, Sanford CG Jr, Patil V, Haman SP, Ren $\mathrm{L}$, et al: Preservation of the spinous process-ligament-muscle complex to prevent kyphotic deformity following laminoplasty. Spine J 7:159-164, 2007

14. Minamide A, Yoshida M, Yamada H, Hashizume H, Nakagawa Y, Nishi H, et al: Efficacy of posterior segmental decompression surgery for pincer mechanism in cervical spondylotic myelopathy: a retrospective case-controlled study using propensity score matching. Spine (Phila Pa 1976) 40:1807-1815, 2015

15. Minamide A, Yoshida M, Yamada H, Nakagawa Y, Maio $\mathrm{K}$, Kawai M, et al: Clinical outcomes of microendoscopic decompression surgery for cervical myelopathy. Eur Spine J 19:487-493, 2010

16. Park JH, Roh SW, Rhim SC, Jeon SR: Long-term outcomes of 2 cervical laminoplasty methods: midline splitting versus unilateral single door. J Spinal Disord Tech 25:E224-E229, 2012

17. Perez-Cruet MJ, Foley KT, Isaacs RE, Rice-Wyllie L, Wellington R, Smith MM, et al: Microendoscopic lumbar discectomy: technical note. Neurosurgery 51 (5 Suppl):S129S136, 2002

18. Ratliff JK, Cooper PR: Cervical laminoplasty: a critical review. J Neurosurg 98 (3 Suppl):230-238, 2003

19. Seichi A, Takeshita K, Ohishi I, Kawaguchi H, Akune T, Anamizu Y, et al: Long-term results of double-door laminoplasty for cervical stenotic myelopathy. Spine (Phila Pa 1976) 26:479-487, 2001

20. Shiraishi T, Fukuda K, Yato Y, Nakamura M, Ikegami T: Results of skip laminectomy-minimum 2-year follow-up study compared with open-door laminoplasty. Spine (Phila Pa 1976) 28:2667-2672, 2003

21. Suk KS, Kim KT, Lee JH, Lee SH, Lim YJ, Kim JS: Sagittal alignment of the cervical spine after the laminoplasty. Spine (Phila Pa 1976) 32:E656-E660, 2007

22. Yamada H, Yoshida M, Hashizume H, Minamide A, Nakagawa Y, Kawai M, et al: Efficacy of novel minimally invasive surgery using spinal microendoscope for treating extraforaminal stenosis at the lumbosacral junction. J Spinal Disord Tech 25:268-276, 2012

23. Yonenobu K, Abumi K, Nagata K, Taketomi E, Ueyama K: Interobserver and intraobserver reliability of the Japanese Orthopaedic Association scoring system for evaluation of cervical compression myelopathy. Spine (Phila Pa 1976) 26:1890-1895, 2001

24. Yoshida M, Tamaki T, Kawakami M, Nakatani N, Ando $\mathrm{M}$, Yamada H, et al: Does reconstruction of posterior ligamentous complex with extensor musculature decrease axial symptoms after cervical laminoplasty? Spine (Phila Pa 1976) 27:1414-1418, 2002

\section{Disclosures}

The authors report no conflict of interest concerning the materials or methods used in this study or the findings specified in this paper.

\section{Author Contributions}

Conception and design: Minamide. Acquisition of data: Minamide, Nakagawa, Okada, Takami. Analysis and interpretation of data: Hashizume, Okada, Nakao. Drafting the article: Yamada, Iwasaki. Critically revising the article: Simpson, Iwasaki. Reviewed submitted version of manuscript: Simpson. Approved the final version of the manuscript on behalf of all authors: Minamide. Statistical analysis: Hashizume. Administrative/technical/ material support: Yamada, Nakagawa, Tsutsui, Takami. Study supervision: Yoshida.

\section{Correspondence}

Akihito Minamide, Department of Orthopaedic Surgery, Wakayama Medical University, 811-1 Kimiidera, Wakayama City, Wakayama 641-8510, Japan. email: minamide@wakayama-med. ac.jp. 